\title{
CARACTERIZACIÓN MORFOLÓGICA Y AGRONÓMICA DE SIETE RAZAS DE MAÍZ DE LOS VALLES ALTOS DE MÉXICO
}

\section{MORPHOLOGICAL AND AGRONOMIC CHARACTERIZATION OF SEVEN MAIZE RACES FROM THE HIGHLANDS OF MÉXICO}

\author{
Mario Rocandio-Rodríguez ${ }^{1}$, Amalio Santacruz-Varela ${ }^{1 \star}$, Leobigildo Córdova-Téllez ${ }^{1}$, Higinio López-Sánchez ${ }^{2}$, \\ Fernando Castillo-González ${ }^{1}$, Ricardo Lobato-Ortiz ${ }^{1}$, J. Jesús García-Zavala ${ }^{1}$ y Rafael Ortega-Paczka ${ }^{3}$
}

\begin{abstract}
${ }^{1}$ Campus Montecillo, Colegio de Postgraduados. Km 36.5 Carretera México-Texcoco. 56230, Montecillo, Texcoco, Edo. de México, México. ${ }^{2}$ Campus Puebla, Colegio de Postgraduados. Km 125.5 Carretera México-Puebla. 72760, Santiago Momoxpan, Puebla, México. ${ }^{3}$ Centros Regionales, Universidad Autónoma Chapingo. Km 38.5 Carretera México-Texcoco. 56230, Chapingo, Texcoco, Edo. de México, México.

*Autor para correspondencia (asvarela@colpos.mx)
\end{abstract}

\section{RESUMEN}

Dentro de la diversidad del maíz (Zea mays L.) en México, uno de los grupos genéticos de mayor interés es el del Complejo Piramidal Mexicano que se cultiva en los Valles Altos Centrales. Los objetivos de este estudio fueron valorar la diversidad morfológica y agronómica en una muestra amplia de poblaciones de las siete razas de maíz más cultivadas en los Valles Altos Centrales de México (Arrocillo Amarillo, Chalqueño, Cónico, Elotes Cónicos, Cacahuacintle, Palomero Toluqueño y Purépecha), y registrar información sobre las características que describen la variación predominante en las razas en estudio. Durante el ciclo agrícola primavera-verano de 2010 se establecieron experimentos con 119 accesiones representativas de estas siete razas de maíz, en Ciudad Serdán y San Mateo Capultitlán, Puebla, y Montecillo, Edo. de México, mediante un diseño experimental látice simple $11 \times 11$. Con las 32 variables cuantitativas medidas se realizó un análisis de varianza y se seleccionaron 13 de ellas, con base en su coeficiente de repetibilidad y coeficientes de correlación, para ejecutar análisis multivariados. El análisis de varianza indicó la existencia de diferencias significativas entre genotipos para todas las variables. La dispersión de accesiones mostró un continuo que se distribuye a lo largo y ancho del plano cartesiano formado por los dos primeros componentes principales, donde influyen en mayor grado las variables de grano relacionadas con tamaño de mazorca y grano (longitud de mazorca, anchura de grano, espesor de grano y la relación anchura de grano/longitud de grano), así como días a floración femenina y número de hojas arriba de la mazorca. Las características menos afectadas por el ambiente fueron las más apropiadas para la agrupación de las accesiones. Los resultados confirmaron los agrupamientos de las accesiones en las razas previamente descritas, así como las interrelaciones entre las mismas de acuerdo con reportes de estudios previos; pero los presentes son más precisos en la definición de los grupos y de las interrelaciones de las razas que en otros estudios, en virtud de los amplios tamaños de muestra utilizados.

Palabras clave: Zea mays, diversidad genética, germoplasma, recursos genéticos.

\section{SUMMARY}

Within the diversity of maize (Zea mays L.) in México, one of the most interesting genetic groups is the Mexican Pyramidal Complex grown in the Central highlands of the country. This study assessed the morphological and agronomic diversity in a large sample of popu- lations of the seven races of maize most commonly cultivated in the highlands of Central México (Arrocillo Amarillo, Chalqueño, Cónico, Elotes Cónicos, Cacahuacintle, Palomero Toluqueño and Purépecha), and to register information of characteristics that describe the prevailing variation of the races under study. Field experiments were established during the 2010 Spring-Summer agricultural season with 119 representative accessions of the aforementioned races of maize, at Ciudad Serdán and San Mateo Capultitlán, Puebla, and at Montecillo, State of México, using an $11 \times 11$ simple lattice experimental design. Thirty-two quantitative traits were recorded and submitted to analysis of variance, and 13 of those traits were selected based on their repeatability and correlation coefficients, for applying multivariate analyses. Analysis of variance showed highly significant differences among genotypes for all the analyzed traits. The dispersion of accessions ended up as a continuum distributed throughout the Cartesian plane formed by the first two principal components, mainly influenced by traits related to ear and kernel size (ear length, kernel width and thickness, and the ratio kernel width/kernel length), as well as days to female flowering and number of leaves above the ear. The traits less affected by the environment were the most appropriate ones for grouping the accessions. Results of this study confirmed the grouping of the accessions of previously described races, as well as the interrelations among them as reported in previous studies; however, our result are more precise than the former studies in defining the groups and the interrelationships among the races because of the large sample sizes used.

Index words: Zea mays L., genetic diversity, germplasm, genetic resources.

\section{INTRODUCCIÓN}

México se caracteriza por presentar condiciones orográficas muy variadas, cuya interacción con los factores climáticos ha generado una amplia diversidad ambiental y nichos ecológicos. De los cultivos cuyo centro de origen y diversidad es México, el maíz (Zea mays L.) es el de mayor importancia a escala nacional y mundial, tanto por la superficie sembrada como por el volumen de producción (FAO, 2009), y su diversidad de usos (CONABIO, 2008).

Para entender su amplitud de adaptación ambiental y características morfológicas apropiadas para diversos usos, 
las poblaciones de maíz se agrupan con base en la categoría de raza. Por décadas varios investigadores han señalado a las variables morfológicas como una herramienta útil para la clasificación racial en maíz (Anderson y Cutler, 1942; Anderson, 1945; Wellhausen et al., 1951; Goodman y Paterniani, 1969; Hernández y Alanís, 1970). Por tanto, para lograr una caracterización racial correcta es necesario conocer de manera detallada la variación existente dentro de las razas (Castillo, 1993), lo que implica valorar la diversidad de poblaciones consideradas como variantes de una raza, con el propósito de diseñar el aprovechamiento de algunas formas dentro de la diversidad genética regional de la especie.

Dentro de la diversidad de maíz de México, uno de los grupos de mayor interés es el denominado “Tipo de México Central" (Kuleshov, 1981), también conocido como Complejo Piramidal Mexicano. Este grupo incluye a las razas: Palomero Toluqueño, Arrocillo Amarillo, Cónico, Elotes Cónicos, Cacahuacintle y Chalqueño, todas ellas cultivadas en Valles Altos, más la raza Purépecha descrita por Mijangos-Cortés et al. (2007).

Los estudios de la diversidad del maíz de la parte central de México han incluido escasas muestras representativas de las razas reportadas en la región (Wellhausen et al., 1951; Ortega y Sánchez, 1989), o bien se han concentrado en una sola raza (Romero et al., 2002; Herrera-Cabrera et al., 2004; Mijangos-Cortés et al., 2007) a mayor parte de los estudios realizados hasta ahora se han hecho desde una perspectiva muy global, abarcando al mismo tiempo todas o la gran mayoría de las razas pero con un número de poblaciones muy limitado de cada una de ellas, dando como resultado una idea muy limitada de la amplitud de la diversidad existente dentro de cada una de las razas; tampoco se han determinado los caracteres más apropiados para la sistematización de la diversidad de estos maíces en particular.

Con base en lo anterior, los objetivos de este trabajo fueron: 1) Analizar la diversidad morfológica y agronómica de las razas de maíz Arrocillo Amarillo, Chalqueño, Cónico, Elotes Cónicos, Cacahuacintle, Palomero Toluqueño y Purépecha cultivadas en los Valles Altos Centrales de México, y 2) Registrar las características que describen la variación predominante en las razas en estudio.

\section{MATERIALES Y MÉTODOS}

\section{Material genético}

Se utilizaron 119 accesiones representativas de siete razas de maíz de los Valles Altos de México (Cuadro 1), las cuales fueron previamente identificadas en campo de manera visual por expertos clasificadores como las que reunían las características de mazorca y planta más afines a la raza. La semilla utilizada se obtuvo de los bancos de germoplasma del Centro Internacional de Mejoramiento de Maíz y Trigo, de la Universidad Autónoma Chapingo, del Instituto Nacional de Investigaciones Forestales, Agrícolas y Pecuarias y del Colegio de Postgraduados.

\section{Ubicación de los experimentos}

Los experimentos fueron establecidos durante el ciclo primavera-verano de 2010 en tres ambientes de los Valles Altos Centrales: Ciudad Serdán ( $18^{\circ} 50^{\prime} \mathrm{N}$ y $97^{\circ} 28^{\prime} \mathrm{O}$, altitud de $2570 \mathrm{~m}$, con promedios anuales de temperatura y precipitación de $14.5^{\circ} \mathrm{C}$ y $521.6 \mathrm{~mm}$ ) y San Mateo Capultitlán ( $19^{\circ} 08^{\prime} \mathrm{N}$; $98^{\circ} 28^{\prime} \mathrm{O}$, altitud de $2260 \mathrm{~m}$, con promedios de temperatura y precipitación de $16.5^{\circ} \mathrm{C}$ y 785.8 $\mathrm{mm})$, ambos sitios en el Estado de Puebla; y en Montecillo, Estado de México (19 $27^{\circ} \mathrm{N}$ y $98^{\circ} 54^{\prime} \mathrm{O}$, altitud de $2250 \mathrm{~m}$, con temperatura y precipitación de $15.1{ }^{\circ} \mathrm{C}$ y $656.9 \mathrm{~mm}$ ). Esta información se obtuvo mediante el programa Eric ${ }^{\circledR}$ III V.2.0 (SMN, 2009).

\section{Conducción de experimentos}

Las siembras se realizaron el 27 de marzo, 23 de abril y 18 de mayo de 2010 en Ciudad Serdán, San Mateo Capultitlán, y Montecillo, respectivamente. Las 119 accesiones se evaluaron en condiciones de temporal (secano), con excepción de Montecillo donde se aplicaron riegos cada vez que lo requirió el cultivo. Se depositaron tres semillas cada $0.50 \mathrm{~m}$ entre matas y $0.80 \mathrm{~m}$ entre surcos. Se hizo un aclareo posterior a la segunda escarda para dejar dos plantas por mata. La fertilización en Ciudad Serdán y San Mateo Capultitlán fue con las dosis $135 \mathrm{~N}-50 \mathrm{P}-00 \mathrm{~K}$, de la cual se aplicó un tercio del nitrógeno y el total del fosforo en la primera escarda, y el resto del nitrógeno en la segunda escarda. En Montecillo se utilizó la fórmula $140 \mathrm{~N}-60 \mathrm{P}-00 \mathrm{~K}$, aplicando $60 \%$ del nitrógeno y todo el fósforo en la siembra y el resto del nitrógeno en la segunda escarda. Las cosechas se efectuaron en diciembre del 2010.

\section{Diseño y unidad experimental}

Se utilizó un diseño experimental látice simple 11 x 11 con dos repeticiones. La unidad experimental consistió de dos surcos de $5 \mathrm{~m}$ de longitud y $0.8 \mathrm{~m}$ de ancho, con una población final de 44 plantas, que corresponden a una densidad de población de 55 mil plantas por hectárea.

\section{Variables evaluadas}

En cada parcela experimental se eligieron al azar cinco plantas con competencia completa, de las cuales se registraron variables fenológicas, vegetativas, de espiga, mazorca y grano. 


\begin{tabular}{lcc}
$\begin{array}{l}\text { Cuadro 1. Razas y número de accesiones de maíz de Valles Altos de México caracterizadas en } \\
\text { Ciudad Serdán y San Mateo Capultitlán, Puebla, y Montecillo Edo. de México, } 2010 .\end{array}$ \\
\hline Raza & Abreviatura & Número de accesiones \\
\hline Arrocillo Amarillo & A & 15 \\
Cacahuacintle & $\mathrm{C}$ & 11 \\
Chalqueño & $\mathrm{Q}$ & 26 \\
Cónico & $\mathrm{N}$ & 24 \\
Elotes Cónicos & $\mathrm{E}$ & 15 \\
Palomero Toluqueño & $\mathrm{T}$ & 8 \\
Purépecha & $\mathrm{P}$ & 20 \\
\hline
\end{tabular}

Las variables de tipo fenológico evaluadas fueron días a floración masculina y femenina, contados a partir de la siembra hasta el momento en que $50 \%$ de las plantas liberaron polen y $50 \%$ de los estigmas estaban expuestos, respectivamente. La asincronía floral se consideró como la diferencia entre la floración masculina y femenina.

Durante el ciclo biológico fueron evaluados los rasgos: número de hijuelos por planta; altura de planta y altura de mazorca, medidos en $\mathrm{cm}$ desde el nivel del suelo hasta la punta de la espiga y hasta el nudo de inserción de la primera mazorca, respectivamente; número total de hojas y número de hojas arriba de la mazorca, contadas al momento de la antesis; longitud y anchura de la hoja de la mazorca principal registradas en $\mathrm{cm}$.

Después de la antesis en la espiga se evaluó: longitud del pedúnculo, medido en $\mathrm{cm}$ desde el nudo superior hasta la primera ramificación; longitud del tramo ramificado de la espiga, medido en $\mathrm{cm}$ desde la primera hasta la última ramificación; longitud de la rama central de la espiga, medido en $\mathrm{cm}$ desde la última ramificación hasta la punta de la espiga; longitud total de la espiga, registrado en $\mathrm{cm}$ desde el nudo superior hasta la punta, y el número de ramificaciones primarias de la espiga.

Al momento de la cosecha se seleccionaron cinco mazorcas para medir en cada una: longitud y el diámetro medio de la mazorca en cm; número de hileras de granos por mazorca; longitud del pedúnculo de la mazorca y diámetro del olote, registrados en $\mathrm{cm}$.

Las cinco mazorcas de cada parcela experimental fueron desgranadas, y se tomaron 10 granos de cada una para medirles en $\mathrm{mm}$ la longitud, anchura y espesor, y se registró el promedio de los 10 granos. Se formó una muestra con 100 granos de las cinco mazorcas y se obtuvo su peso; los mismos 100 granos se colocaron en una probeta y se midió el volumen en $\mathrm{cm}^{3}$; el porcentaje de grano, se obtuvo del cociente peso total del grano de la mazorca/peso total de la mazorca $\times 100$. De los datos obtenidos se estimaron seis relaciones: de la parte vegetativa (altura de mazorca/altura de planta); de la espiga (longitud del tramo ramificado de la espiga/longitud total de la espiga); de la mazorca (diámetro medio de la mazorca/longitud), y del grano (anchura/ longitud, espesor/longitud y peso de 100 granos/volumen de 100 granos).

\section{Análisis estadístico}

Con el empleo de los promedios de cada unidad experimental de las tres localidades para las 32 variables, y como no se encontró eficiencia en el diseño de látice, se corrió un análisis de varianza en bloques completos al azar ordinarios combinado bajo este diseño, mediante el paquete $S A S \AA$ V.9.0. (SAS Institute, 2002), a fin de estimar la interacción genotipo $\times$ ambiente; además, con el mismo paquete se obtuvieron los componentes de varianza a través de las esperanzas de cuadrados medios (Cuadro 2). La estimación de los componentes de varianza se obtuvo como:

$$
\begin{aligned}
& \sigma_{g}^{2}=[C M G-C M G \times L] / r l \\
& \sigma_{l}^{2}=[C M L-C M R L] / r g \\
& \sigma_{g x l}^{2}=[C M G \times L-C M E] / r
\end{aligned}
$$

Se hizo una selección de variables a través de dos métodos de discriminación (Sánchez et al., 1993; Herrera-Cabrera et al., 2000): 1) Por el criterio de Goodman y Paterniani (1969), se seleccionaron aquéllas con un coeficiente de repetibilidad $\mathrm{r}=\left[\sigma_{g}^{2} /\left(\sigma_{l}^{2}+\sigma_{g x l}^{2}\right)\right] \geq 1$, donde $\sigma_{g}^{2}$ y $\sigma_{l}^{2}$ son estimadores de los componentes de varianza de genotipos y localidades, respectivamente, y $\sigma_{g x l}^{2}$ es el estimador del componente de varianza de la interacción entre genotipos y localidades; y 2) Mediante la obtención de valores y vectores propios a partir de la matriz de correlaciones (Rawlings, 1988), junto con una gráfica de Gabriel (Gabriel, 1971) para generar una representación visual de la estructura de correlaciones entre variables y la magnitud de cada variable sobre la varianza global de los datos (Pla, 1986). 
Cuadro 2. Análisis de varianza combinado.

\begin{tabular}{|c|c|c|c|}
\hline Fuente de variación & Grados de Libertad & Cuadrados Medios & $\begin{array}{c}\text { Esperanzas de cuadrados } \\
\text { medios }\end{array}$ \\
\hline Localidades (Loc) & $l-1$ & CML & $\sigma_{e}^{2}+g \sigma_{r(l)}^{2}+r g \sigma_{l}^{2}$ \\
\hline Rep/Loc & $l(\mathrm{r}-1)$ & CMRL & $\sigma_{e}^{2}+g \sigma_{r(l)}^{2}$ \\
\hline Genotipos (Gen) & g-1 & CMG & $\sigma_{e}^{2}+r \sigma_{g \times l}^{2}+l r \sigma_{g}^{2}$ \\
\hline Gen x Loc & $(g-1)(l-1)$ & $\mathrm{CMG} \times \mathrm{L}$ & $\sigma_{e}^{2}+r \sigma_{g \times l}^{2}$ \\
\hline Error & $l(\mathrm{r}-1)(\mathrm{g}-1)$ & CME & $\sigma_{e}^{2}$ \\
\hline Total & $\mathrm{rgl}-1$ & & \\
\hline
\end{tabular}

l: número de localidades; r: número de repeticiones en cada localidad; g: número de genotipos; $\sigma_{e}^{2}=$ varianza del error; $\sigma_{r(l)}^{2}=$ varianza de la repetición anidada a la localidad; $\sigma_{g}^{2}=$ varianza genética; $\sigma_{l}^{2}=$ varianza de localidades; $\sigma_{g x l}^{2}=$ varianza de la interacción genotipo por localidad.

Además se hizo un análisis de componentes principales, con base en la matriz de correlaciones entre las variables seleccionadas, las cuales fueron analizadas con el método no paramétrico de agrupación MODECLUS, el cual se utiliza para estimar un número adecuado de agrupaciones en las que la cantidad de elementos de cada grupo se basa en un valor de partida (kernel) que corresponde al radio (R) de una esfera multidimensional expresado en términos de distancia euclidiana, sobre la cual se explora el número de elementos que caen dentro de la hiper-esfera con un alcance determinado por R (SAS Institute, 2002).

También se hizo un análisis de conglomerados con datos estandarizados a la distribución normal $(0,1)$, mediante las distancias euclidianas y el método de ligamiento promedio (UPGMA). La altura de corte para definir los grupos se determinó con base en el índice Pseudo F. Estos análisis se efectuaron con los paquetes estadísticos SAS® V.9.0. (SAS Institute, 2002) y NTSYSpc® V.2.21 (Rohlf, 2009).

\section{RESULTADOS Y DISCUSIÓN}

\section{Análisis de varianza}

Se detectó un alto grado de variación entre los genotipos para todas las variables evaluadas, por lo que se infiere la existencia de una gran diversidad genética entre los mismos. Entre los ambientes hubo diferencias significativas ( $P$ $\leq 0.01$ ) en 27 de los 32 atributos morfológicos (Cuadro 3). Con tales diferencias se demuestra considerable contraste entre las localidades de cultivo, en la cual influyeron, además de las características climáticas y edáficas de las localidades, las diferencias en fechas de siembra y en manejo del cultivo en cada localidad.

La interacción genotipo $\times$ ambiente resultó significativa $(\mathrm{P} \leq 0.01)$ para la mayoría de las variables, excepto para: altura de planta, longitud del pedúnculo de la espiga, longitud del tramo ramificado de la espiga, longitud de la ma- zorca, anchura de grano, porcentaje de grano de la mazorca, peso de 100 granos, volumen de 100 granos, espesor/longitud de grano y peso/volumen de 100 granos; nuevamente, las diferencias muestran que los genotipos tienen una respuesta particular en cada una de las localidades del estudio (Cuadro 3).

\section{Selección de variables}

Los estimadores de los componentes de varianza para genotipos $\left(\sigma_{g}^{2}\right)$, localidades $\left(\sigma_{l}^{2}\right)$, interacción genotipos $\times$ localidades $\left(\sigma_{g x l}^{2}\right)$ y repetibilidad $\mathrm{r}=\left[\sigma_{g}^{2} /\left(\sigma^{2}{ }_{l}+\sigma_{g x l}^{2}\right)\right]$ de las 32 variables, mostraron valores de $r \geq 1$ para 19 atributos morfológicos (Cuadro 4), las cuales fueron seleccionadas por su estabilidad a través de localidades en una primera etapa. Con excepción de altura de planta y longitud del tramo ramificado de la espiga, las variables seleccionadas en esta etapa no presentaron interacción genotipos $x$ localidades en los análisis de varianza (Cuadro 3), lo que les confiere propiedades deseables para la clasificación, pues de acuerdo con Sánchez et al. (1993) los atributos morfológicos que son menos afectados por el ambiente son más útiles para caracterizar a las poblaciones.

Algunas de las variables detectadas aquí como apropiadas para la clasificación, también fueron seleccionadas en otros estudios con criterios similares; así Sánchez et al. (1993) indican que las características de la mazorca o reproductivas, entre los que se incluye a número de hojas totales, longitud de la rama central de la espiga, anchura de grano, diámetro/longitud de mazorca y anchura/longitud de grano, son las más apropiadas para la caracterización racial en maíz. En una segunda etapa para definir el conjunto final de variables se analizó la matriz de correlaciones y gráficas de Gabriel para detectar problemas de colinealidad, lo que permitió eliminar las variables cuyo coeficiente de correlación fuera mayor de 0.7 .

De esta manera se definieron 13 variables (días a floración 
Cuadro 3. Cuadrados medios del análisis de varianza combinado a través de localidades.

\begin{tabular}{|c|c|c|c|c|}
\hline Variable & Genotipos & Localidades & Gen $\times$ Loc & Error \\
\hline Floración femenina (días) & $932.5^{\star *}$ & $2.6 \times 10^{4 * *}$ & $39.4^{\star *}$ & 13.5 \\
\hline Floración masculina (días) & $813.2^{\star *}$ & $3.4 \times 10^{4 * *}$ & $33.0^{* *}$ & 15.7 \\
\hline Asincronia floral (días) & $33.5^{\star *}$ & $659.8^{* *}$ & $9.9^{* *}$ & 5.5 \\
\hline Hijuelos por planta (numérico) & $0.1^{* *}$ & $15.03^{* *}$ & $0.07^{\star *}$ & 0.1 \\
\hline Altura de planta $(\mathrm{cm})$ & $1.0 \times 10^{4 * *}$ & $4.2 \times 10^{5 * *}$ & 330.9 ns & 288.2 \\
\hline Altura de mazorca $(\mathrm{cm})$ & $5441.2^{* *}$ & $3.2 \times 10^{5 * *}$ & $254.4^{* *}$ & 206.4 \\
\hline Altura de mazorca/Altura de planta & $0.1^{\star *}$ & $1.2^{\star *}$ & $0.003^{\star *}$ & 0.002 \\
\hline Hojas totales (numérico) & $21.5^{\star *}$ & $25.4 \mathrm{~ns}$ & $1.2^{\star *}$ & 0.6 \\
\hline Hojas arriba de la mazorca (numérico) & $2.1^{\star *}$ & $10.6^{* *}$ & $0.1^{* *}$ & 0.1 \\
\hline Longitud de la hoja de la mazorca $(\mathrm{cm})$ & $393.6^{* *}$ & $5.7 \times 10^{4 * *}$ & $57.5^{\star *}$ & 29.2 \\
\hline Anchura de la hoja de la mazorca $(\mathrm{cm})$ & $7.6^{* *}$ & $955.6^{* *}$ & $1.7^{\star}$ & 1.4 \\
\hline Longitud de la espiga $(\mathrm{cm})$ & $155.3^{\star *}$ & $210.7 \mathrm{~ns}$ & $30.0^{* *}$ & 25.6 \\
\hline Longitud pedúnculo de la espiga (cm) & $30.6^{* *}$ & $248.5 \mathrm{~ns}$ & $12.9 \mathrm{~ns}$ & 11.1 \\
\hline Longitud rama central de la espiga $(\mathrm{cm})$ & $64.6^{\star *}$ & $264.9^{* *}$ & $10.6^{\star *}$ & 7.7 \\
\hline Longitud tramo ramificado de la espiga $(\mathrm{cm})$ & $23.6^{\star *}$ & $1190.5^{\star *}$ & $2.4 \mathrm{~ns}$ & 2.2 \\
\hline $\begin{array}{l}\text { Longitud del tramo ramificado/Longitud de } \\
\text { la espiga }\end{array}$ & $0.005^{\star *}$ & $0.3^{* *}$ & $0.001^{* *}$ & 0.001 \\
\hline Ramas primarias de la espiga (numérico) & $38.2^{\star *}$ & $1180.2^{* *}$ & $6.2^{* *}$ & 4.8 \\
\hline Longitud de la mazorca $(\mathrm{cm})$ & $34.8^{\star *}$ & $423.7^{\star *}$ & $2.9 \mathrm{~ns}$ & 2.7 \\
\hline Hileras de la mazorca (numérico) & $24.9^{* *}$ & $186.7^{* *}$ & $1.3^{* *}$ & 1.0 \\
\hline Longitud pedúnculo de la mazorca $(\mathrm{cm})$ & $26.1^{\star *}$ & $1549.9^{* *}$ & $8.5^{\star *}$ & 6.5 \\
\hline Diámetro de la mazorca $(\mathrm{cm})$ & $0.9^{\star *}$ & $34.6^{* *}$ & $0.1^{\star *}$ & 0.1 \\
\hline Diámetro/Longitud de la mazorca & $0.01^{\star *}$ & $0.03 \mathrm{~ns}$ & $0.001^{\star *}$ & 0.001 \\
\hline Diámetro de olote $(\mathrm{cm})$ & $0.6^{* *}$ & $12.3^{\star *}$ & $0.1^{\star *}$ & 0.1 \\
\hline Longitud de grano (mm) & $783.1^{\star *}$ & $1.4 \times 10^{4 \star *}$ & $97.8^{\star *}$ & 64.1 \\
\hline Anchura de grano (mm) & $826.7^{\star *}$ & $1500.4^{* *}$ & $20.9 \mathrm{~ns}$ & 18.1 \\
\hline Espesor de grano (mm) & $156.9^{* *}$ & $138.3^{* *}$ & $9.7^{* *}$ & 7.3 \\
\hline Porcentaje de grano de la mazorca (\%) & $56.6^{* *}$ & $223.1^{* *}$ & $14.4 \mathrm{~ns}$ & 14.7 \\
\hline Peso de 100 granos $(\mathrm{g})$ & $248.1^{\star *}$ & $1114.0^{* *}$ & $22.2 \mathrm{~ns}$ & 18.8 \\
\hline Volumen de 100 granos $\left(\mathrm{cm}^{3}\right)$ & $811.2^{* *}$ & $2990.6^{* *}$ & $50.1 \mathrm{~ns}$ & 47.1 \\
\hline Anchura de grano/Longitud de grano & $0.05^{\star *}$ & $0.1^{* *}$ & $0.004^{\star *}$ & 0.003 \\
\hline Espesor de grano/Longitud de grano & $0.02^{* *}$ & $0.1^{\star *}$ & $0.003 \mathrm{~ns}$ & 0.002 \\
\hline Peso de 100 granos/Volumen de 100 granos & $0.01^{* *}$ & $0.02 \mathrm{~ns}$ & $0.002 \mathrm{~ns}$ & 0.002 \\
\hline
\end{tabular}

${ }^{*}$ Significancia a $5 \%$; ${ }^{* *}$ significancia a $1 \%$; ns = no significativo.

femenina, longitud de la rama central de la espiga, hojas arriba de la mazorca, número de hileras de la mazorca, longitud de la mazorca, diámetro/longitud de la mazorca, anchura de grano, peso de 100 granos, anchura/longitud de grano, espesor/longitud de grano, espesor de grano, longitud de grano y peso/volumen de 100 granos), con las cuales pudo obtenerse información de calidad para valorar la diversidad racial en el grupo de poblaciones de maíz de los Valles Altos de México.

Algunas de estas variables también han sido seleccionados en otros estudios de diversidad genética del maíz (Sánchez et al., 1993; Herrera-Cabrera et al., 2000; Sánchez et al., 2000; López-Romero et al., 2005; Mijangos-Cortés et 
Cuadro 4. Componentes de varianza para genotipos $\left(\sigma_{g}^{2}\right)$, localidades $\left(\sigma_{l}^{2}\right)$, interacción genotipos $x$ localidades $\left(\sigma_{g x l}^{2}\right)$ y repetibilidad (r) de las variables estudiadas.

\begin{tabular}{|c|c|c|c|c|}
\hline Variable & $\sigma_{g}^{2}$ & $\sigma_{l}^{2}$ & $\sigma_{g \times l}^{2}$ & $\mathrm{r}$ \\
\hline Floración femenina (días) & 148.8 & 108.4 & 12.9 & $1.2^{9}$ \\
\hline Floración masculina (días) & 130.1 & 143.2 & 8.6 & 0.9 \\
\hline Asincronia floral (días) & 3.9 & 2.7 & 2.2 & 0.8 \\
\hline Hijuelos por planta (numérico) & 0.001 & 0.1 & 0.01 & 0.01 \\
\hline Altura de planta $(\mathrm{cm})$ & 1687.5 & 1734.8 & 21.3 & 0.9 \\
\hline Altura de mazorca $(\mathrm{cm})$ & 864.5 & 1346.0 & 24.1 & 0.6 \\
\hline Altura de mazorca/Altura de planta & 0.002 & 0.005 & 0.001 & 0.4 \\
\hline Hojas totales (numérico) & 3.4 & 0.1 & 0.3 & $9.4^{9}$ \\
\hline Hojas arriba de la mazorca (numérico) & 0.3 & 0.1 & 0.02 & 5.39 \\
\hline Longitud de la hoja de la mazorca $(\mathrm{cm})$ & 56.1 & 237.4 & 14.1 & 0.2 \\
\hline Anchura de la hoja de la mazorca $(\mathrm{cm})$ & 0.9 & 3.9 & 0.1 & 0.2 \\
\hline Longitud de la espiga $(\mathrm{cm})$ & 20.9 & 0.2 & 2.2 & $8.6^{9}$ \\
\hline Longitud pedúnculo de la espiga $(\mathrm{cm})$ & 2.9 & 0.9 & 0.9 & $1.7^{9}$ \\
\hline Longitud rama central de la espiga $(\mathrm{cm})$ & 9.1 & 0.9 & 1.4 & $3.7^{9}$ \\
\hline $\begin{array}{l}\text { Longitud tramo ramificado de la espiga } \\
(\mathrm{cm})\end{array}$ & 3.5 & 4.9 & 0.1 & 0.7 \\
\hline $\begin{array}{l}\text { Longitud del tramo ramificado/Longitud } \\
\text { de la espiga }\end{array}$ & 0.001 & 0.001 & 0.0001 & 0.6 \\
\hline Ramas primarias de la espiga (numérico) & 5.3 & 4.9 & 0.7 & 0.9 \\
\hline Longitud de la mazorca $(\mathrm{cm})$ & 5.3 & 1.7 & 0.1 & $2.9^{9}$ \\
\hline Hileras de la mazorca (numérico) & 3.9 & 0.8 & 0.1 & $4.4^{9}$ \\
\hline Longitud pedúnculo de la mazorca $(\mathrm{cm})$ & 2.9 & 6.3 & 1.1 & 0.4 \\
\hline Diámetro de la mazorca $(\mathrm{cm})$ & 0.1 & 0.1 & 0.01 & 0.9 \\
\hline Diámetro/Longitud de la mazorca & $1.0 \times 10^{-3}$ & $01.0 \times 10^{-4}$ & $1.0 \times 10^{-4}$ & $8.1^{9}$ \\
\hline Diámetro de olote $(\mathrm{cm})$ & 0.09 & 0.1 & 0.009 & $1.4^{9}$ \\
\hline Longitud de grano (mm) & 114.2 & 60.0 & 16.9 & $1.5^{9}$ \\
\hline Anchura de grano (mm) & 134.3 & 6.2 & 0.9 & $18.8^{9}$ \\
\hline Espesor de grano $(\mathrm{mm})$ & 24.5 & 0.5 & 1.2 & $14.4^{9}$ \\
\hline Porcentaje de grano de la mazorca (\%) & 7.02 & 0.9 & -0.1 & $9.1^{9}$ \\
\hline Peso de 100 granos $(\mathrm{g})$ & 37.6 & 4.4 & 1.7 & $6.1^{9}$ \\
\hline Volumen de 100 granos $\left(\mathrm{cm}^{3}\right)$ & 126.8 & 12.1 & 1.5 & $9.3^{9}$ \\
\hline Anchura de grano/Longitud de grano & 0.01 & 0.001 & 0.001 & $9.2^{9}$ \\
\hline Espesor de grano/Longitud de grano & $1.0 \times 10^{-3}$ & $1.0 \times 10^{-3}$ & $2.0 \times 10^{-4}$ & $3.1^{9}$ \\
\hline $\begin{array}{l}\text { Peso de } 100 \text { granos/Volumen de } 100 \\
\text { granos }\end{array}$ & $1.0 \times 10^{-3}$ & $1.0 \times 10^{-4}$ & $1.0 \times 10^{-4}$ & $10.2^{9}$ \\
\hline
\end{tabular}

'Variables seleccionadas con base en su repetibilidad ( $\mathrm{r} \geq 1)$. 
al., 2007), lo que confirma su utilidad para medir o diferenciar la diversidad de determinado grupo de poblaciones, entre razas, y entre poblaciones de la misma raza (Herrera-Cabrera et al., 2000; Sánchez et al., 1993). Es necesario mencionar que en la literatura se aborda la definición de un grupo de variables que en su momento se consideraron como "apropiadas" para la sistematización de poblaciones al considerar a todas las razas mexicanas de maíz en su conjunto; sin embargo, a medida que se profundiza en este tipo de estudios se ha observado que las variables morfológicas y agronómicas más apropiadas son diferentes para cada grupo de razas y tiene que ser determinado para cada caso específico.

\section{Análisis de componentes principales}

Con las 13 variables finalmente seleccionadas, los genotipos se sometieron a un análisis de componentes principales (CP). Los dos primeros componentes explicaron 48.5 y 19.7 $\%$ de la variación total, respectivamente (Cuadro 5). En el CP1 la longitud de la rama central de la espiga, longitud de la mazorca, anchura de grano, espesor de grano, peso de 100 granos y la relación anchura/longitud de grano contribuyeron mayormente a la variación explicada por este componente, en tanto que en el CP2 las características de mayor importancia fueron días a floración femenina, hojas arriba de la mazorca y longitud de grano.

La alta proporción de la varianza explicada con un bajo número de componentes permitió una mejor interpretación de esa variación con este tipo de análisis (Pla, 1986). Con base en el análisis de agrupamiento de MODECLUS (SAS Institute, 2002) y con un radio en la hiper-esfera de exploración $\mathrm{R}=0.9$, la dispersión de las accesiones en el plano de los dos primeros CP mostró cinco grupos bien definidos por sus características de grano, mazorca, espiga y duración de su ciclo biológico (Figura 1).

El Grupo I, ubicado en la parte central del Cuadrante IV, se integró por ocho accesiones de la raza Cacahuacintle y por la accesión 7202 de la raza Chalqueño; este grupo destacó por su grano grande y mayor anchura de grano $(9.4$ y $9.3 \mathrm{~mm}$ ), espesor de grano ( 4.8 y $4.6 \mathrm{~mm}$ ) y peso de 100 granos ( $35 \mathrm{y} 45 \mathrm{~g}$ ), con valores similares a los reportados previamente por Herrera-Cabrera et al. (2004).

El Grupo II, disperso en el Cuadrante I, se conformó por 19 accesiones de la raza Purépecha, además de las accesiones Pue-129 de la raza Arrocillo, Mich-86 de la raza Elotes Cónicos y la accesión 186 de la raza Cónico. Este grupo se integró por las accesiones más tardías ( 98.8 a $134.5 \mathrm{~d}$ ), con mazorcas largas ( 16.4 a $20.5 \mathrm{~cm}$ ), longitudes de la rama central de la espiga de 29.5 a $39 \mathrm{~cm}$, y relación anchura/longitud de grano de 0.6 a 0.8 .
Aun cuando mostraron cierta cercanía con la raza Chalqueño, sus características sugieren que estas poblaciones constituyeron un grupo genético diferente de Chalqueño, como fue sugerido por Romero et al. (2002) y después confirmado por Mijangos-Cortés et al. (2007). Las accesiones 186 (de Teloloapan, Guerrero) y Mich-86 (de Jericó de las Flores, Michoacán) se integraron dentro de este grupo, por afinidad en procedencia y distribución con la raza Purépecha, en tanto que Pue-129 se asemejó a la raza Chalqueño por sus características de mazorca y altura de planta.

El Grupo III lo conformaron muestras de las razas Arrocillo y Palomero Toluqueño, y se ubicó en los Cuadrantes II y III, ya que mostraron similitud en características de longitud de mazorca ( 9 a 15.9 y 10.6 a $14.2 \mathrm{~cm}$ ), longitud de la rama central de la espiga ( 22 a 32.1 y 23.9 a $31.8 \mathrm{~cm}$ ) y anchura de grano ( 5.3 a 7.7 y 4.7 a $6.3 \mathrm{~mm}$ ), en concordancia con lo señalado por Wellhausen et al. (1951), quienes mencionaron que estas razas tienen un parentesco muy estrecho que se refleja en casi todas las características de mazorca.

El Grupo IV se distribuyó en los Cuadrantes I y II está integrado por accesiones de las razas Cónico y Chalqueño, en el que éste último mostró una ligera tendencia hacia el Grupo II donde se ubicaron los maíces más tardíos y de mayor tamaño de mazorca. En el Grupo V se encontraron accesiones de las razas Elotes Cónicos, Cónico y Chalqueño, distribuidas en los Cuadrantes III y IV. Según Wellhausen et al. (1951), las razas Cónico y Chalqueño tienen una distribución geográfica casi idéntica en la Mesa Central. Por su parte, el Complejo Piramidal Mexicano presenta dos tipos de patrones varietales y tamaño de mazorca contrastantes, y en la Figura 1 se observa que los más tardíos y de mazorca grande compartieron el Cuadrante I y IV con los Grupos II y I, respectivamente; en contraparte, los más precoces y con mazorca de menor tamaño se distribuyeron en los Cuadrantes II y III.

Tal dispersión puede explicarse al considerar que el Complejo Piramidal Mexicano guarda relaciones y semejanzas con otras razas como Cacahuacintle y Palomero Toluqueño (Wellhausen et al., 1951; Doebley et al., 1985; Sánchez y Goodman, 1992). Las accesiones de las razas Elotes Cónicos y Cónico se ubicaron en torno al origen; tal convergencia es congruente con lo propuesto por Wellhausen et al. (1951), en el sentido a que Elotes Cónicos corresponde a una sub-raza de Cónico.

\section{Análisis de conglomerados}

En el análisis de conglomerados se observó continuidad en los agrupamientos de acuerdo con las medidas de similitud, debido principalmente a la diversidad en la duración del ciclo biológico y tamaño de mazorca (Sánchez et al., 1993; 


\begin{tabular}{|c|c|c|}
\hline Variables & $\mathrm{CP} 1$ & $\mathrm{CP} 2$ \\
\hline Floración femenina & 0.152 & 0.457 \\
\hline Hojas arriba de la mazorca & 0.185 & 0.415 \\
\hline Longitud rama central de la espiga & 0.300 & 0.110 \\
\hline Hileras de la mazorca & -0.268 & 0.191 \\
\hline Longitud de la mazorca & 0.334 & 0.225 \\
\hline Diámetro/Longitud de la mazorca & -0.257 & -0.349 \\
\hline Longitud de grano & 0.036 & -0.381 \\
\hline Anchura de grano & 0.355 & -0.249 \\
\hline Espesor de grano & 0.346 & -0.193 \\
\hline Peso de 100 granos & 0.293 & -0.343 \\
\hline Anchura de grano/Longitud de grano & 0.350 & -0.014 \\
\hline Espesor de grano/Longitud de grano & 0.290 & 0.085 \\
\hline Peso de 100 granos/Volumen de 100 granos & -0.253 & 0.173 \\
\hline Valor propio & 6.3 & 2.5 \\
\hline Varianza explicada (\%) & 48.5 & 19.7 \\
\hline
\end{tabular}

Herrera-Cabrera et al., 2000; Sánchez et al., 2000; HerreraCabrera et al., 2004). En la parte superior del dendrograma se ubicaron las accesiones de ciclo tardío y mazorca grande, y en la parte inferior estuvieron los genotipos con mazorcas pequeñas y más precoces. El dendrograma permitió distinguir tres grupos a una distancia euclidiana de 0.59 , de acuerdo con el procedimiento estadístico de Pseudo F (Figura 2). En el Grupo I se observan dos subgrupos: el Subgrupo I-A que aglomeró las accesiones más tardías (121 a 134 d) y en las que predominaron mazorcas de mayor longitud; de ellas ocho muestras son de la raza Purépecha, ocho de Chalqueño, dos de Cónicos y una de Elotes Cónicos. El Subgrupo I-B congregó a nueve poblaciones de la raza Purépecha y una de Elotes Cónicos, que fueron similares en precocidad (102 a 113 d a floración femenina), mazorca alargada (16.4 a $20.4 \mathrm{~cm}$ ) y semilla grande $(31.2 \mathrm{~g} / 100$ granos).

Las accesiones evaluadas de la raza Purépecha se dividieron en dos subgrupos, uno de ellos se ubicó en el grupo I-A más tardío, y el segundo en el grupo I-B, separados por un conglomerado de Chalqueños, lo que ya había sido observado por Ortega y Sánchez (1989), quienes propusieron la existencia de un nuevo grupo bajo el nombre de "Tarasco". Este conglomerado de maíces Chalqueños albergó poblaciones de esta raza, provenientes de lugares diferentes al Valle de México (Veracruz, Zacatecas, Durango, Morelos, Jalisco, Oaxaca, Querétaro, Guanajuato); es decir, son poblaciones que han experimentado divergencia con respecto al núcleo principal de los Chalqueños y su nicho de adapta- ción se ubica en pequeñas áreas de humedad residual en los estados mencionados. Por su diferenciación marcada del núcleo principal de los Chalqueños, este grupo de maíces donde se encuentran los maíces de Cajete, merece un estudio especial más a fondo.

De acuerdo con los resultados de este estudio, existe la posibilidad de que las muestras más precoces de la raza $\mathrm{Pu}$ répecha formen parte de la raza Chalqueño y el resto sean las que constituyen a la raza Purépecha. En el Grupo II también hubo dos Subgrupos: el Subgrupo II-A, formado por las razas Cónico y Elotes Cónicos, de semilla mediana y de mayor precocidad, y el Subgrupo II-B compuesto mayormente por accesiones de la raza Cacahuacintle, de 88 a 106 $\mathrm{d}$ a floración femenina y semilla grande (35 g/100 granos). El Grupo III se integró por las accesiones de las razas Palomero Toluqueño y Arrocillo Amarillo, de semilla pequeña (16 a $20 \mathrm{~g} / 100$ granos), mayor cantidad de hileras por mazorca (16.3 a 17.2) y de ciclo biológico corto (83.4 a $93.5 \mathrm{~d}$ a floración femenina).

\section{CONCLUSIONES}

Dentro del Complejo Piramidal Mexicano, las razas indígenas antiguas Palomero Toluqueño y Arrocillo Amarillo se diferencian claramente del resto, mientras que la raza Chalqueño se diferencia de la raza Cónico por ser más tardía y de mayor tamaño de mazorca ( 100 y 88.5 d a floración masculina; $15.6 \mathrm{~cm}$ y $13 \mathrm{~cm}$ de longitud de mazorca, 


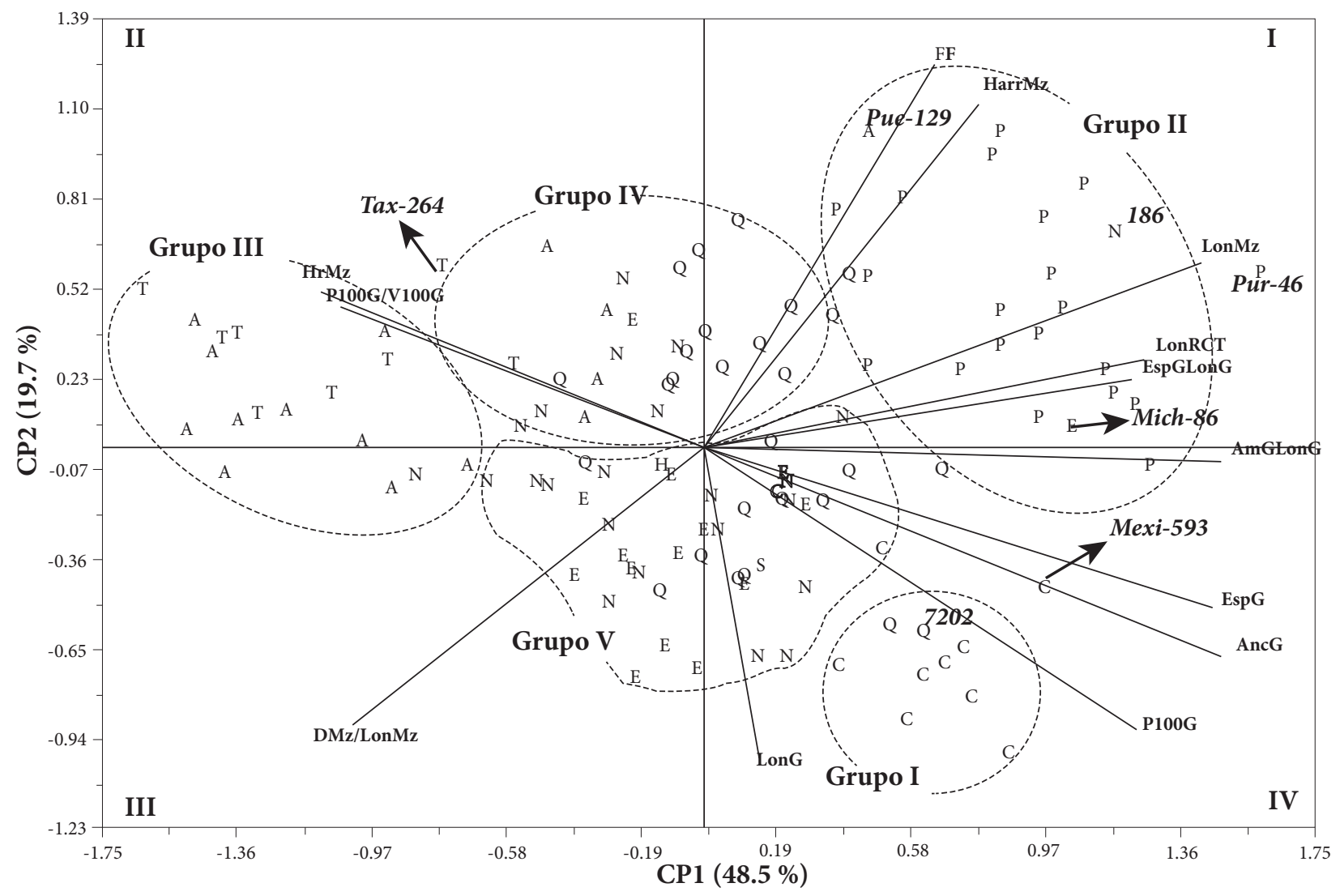

Figura 1. Dispersión de 119 accesiones de maíz de los Valles Altos de México con base en los dos primeros componentes principales. $\mathrm{P}=$ purépecha; $\mathrm{C}=$ cacahuacintle; $\mathrm{E}=$ elotes cónicos; $\mathrm{N}=$ cónico; $\mathrm{T}=$ palomero toluqueño; $\mathrm{Q}=$ chalqueños; $\mathrm{A}=$ arrocillo amarillo; $\mathrm{FF}$ = floración femenina; $\mathrm{HarrMz}$ = hojas arriba de la mazorca; $\mathrm{LonMz}$ = longitud de la mazorca; LonRCE = longitud de la rama central de la mazorca; EspG/LonG = relación espesor/longitud de grano; $\mathrm{AncG} / \mathrm{LonG}=$ relación anchura/longitud de grano; EspG = espesor de grano; AncG = anchura de grano; $P 100 G$ = peso de 100 granos; LonG = longitud de grano; $\mathrm{DMz} / \mathrm{LonMz}$ = diámetro/longitud de la mazorca; $\mathrm{HiMz}=$ hileras de grano por mazorca; $\mathrm{P} 100 \mathrm{G} / \mathrm{V} 100 \mathrm{G}=$ peso/ volumen de cien granos.

respectivamente).

En el análisis de conglomerados una parte de las muestras de la raza Purépecha se separaron considerablemente de la raza Chalqueño, por su mayor longitud y menor diámetro de mazorca, así como por su ciclo biológico más tardío, lo que permite suponer que constituyen un grupo independiente, mientras que otra parte de las muestras de esa raza fueron más afines a Chalqueño lo que muestra que estas dos razas están aún en proceso de divergencia a tal grado que una parte de los Purépechas en realidad son todavía Chalqueños, o que hay que reconocer dos razas diferentes en lugar de una sola. Se recomienda que se estudie más profundamente la diversidad genética de esta raza mediante herramientas moleculares.

Se definieron las variables días a floración femenina, longitud de la rama central de la espiga, hojas arriba de la mazorca, número de hileras de la mazorca, longitud de la mazorca, diámetro/longitud de la mazorca, anchura de grano, peso de 100 granos, anchura/longitud de grano, espesor/longitud de grano, espesor de grano, longitud de grano y peso/volumen de 100 granos, como las más apropiadas para la caracterización racial en maíces de Valles Altos de México.

La inclusión de un considerable número de muestras indujo certidumbre en la diferenciación morfológica y agronómica de los diferentes tipos de maíz de los Valles Altos Centrales. Así mismo, se confirmó la existencia e identidad de las razas previamente reportadas, al igual que los agrupamientos de las accesiones y las interrelaciones entre las razas. 


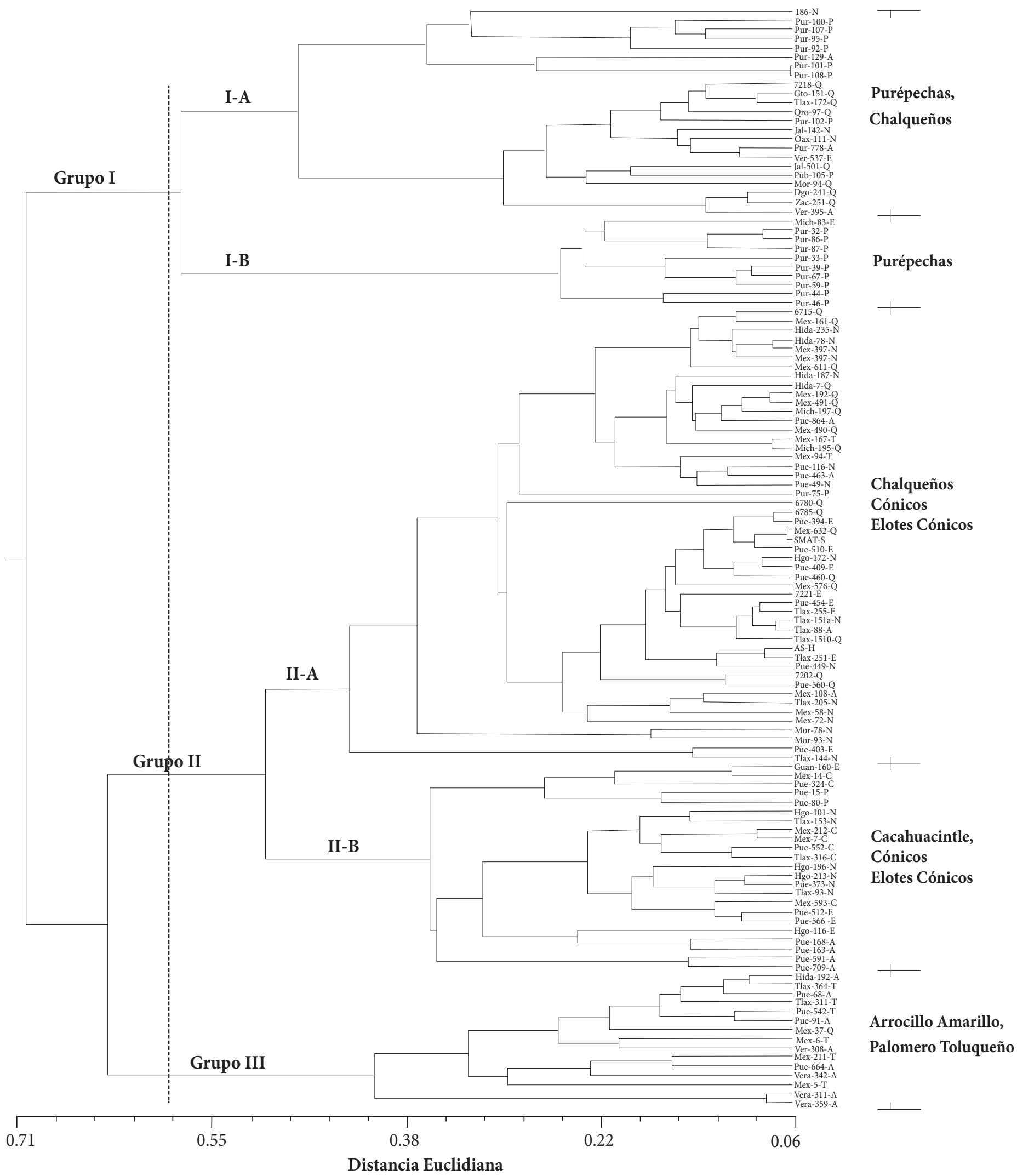

Figura 2. Dendrograma de 119 accesiones de maíz de Valles Altos de México, construido mediante UPGMA con distancias euclidianas derivadas de 13 variables morfológicas. 


\section{BIBLIOGRAFÍA}

Anderson E. (1945) Maize in the new world. In: New Crops in the New World. C. M. Wilson (ed). McMillan Co. New York. pp:27-42.

Anderson E. and H. C. Cutler (1942) Races of Zea mays: I. Their recognition and classification. Annals of the Missouri Botanical Garden 29:69-88.

Castillo G. F. (1993) La variabilidad genética y el mejoramiento genético de los cultivos. Ciencia 44:69-79.

CONABIO, Comisión Nacional para el Conocimiento y Uso de la Biodiversidad (2008) Capital Natural de México, Volumen I: Conocimiento Actual de la Biodiversidad. Comisión Nacional para el Conocimiento y Uso de la Biodiversidad, México. Disponible en: www.conabio.gob.mx, (Noviembre 2013).

Doebley J. F., M. M. Goodman and C. W. Stuber (1985) Isozyme variation in the races of maize from Mexico. American Journal of Botany 72:629-639.

FAO, Food and Agriculture Organization (2009) Base de Datos de Estadísticas Agropecuarias. Disponible en: www.faostat.fao.org (Noviembre 2013).

Gabriel K. (1971) The biplot graphic display of matrices with application to principal component analysis. Biometrika 58:453-467.

Goodman M. M. and E. Paterniani (1969) The races of maize. III. Choices of appropriate characters for racial classification. Economic Botany 23:265-273.

Hernández X. E. y G. Alanís F. (1970) Estudio morfológico de cinco nuevas razas de maíz de la Sierra Madre Occidental de México- implicaciones filogenéticas y fitogeográficas. Agrociencia 5:3-30.

Herrera-Cabrera B. E., F. Castillo-González, J. J. Sánchez-González, R. Ortega-Paczka y M. M. Goodman (2000) Caracteres morfológicos para valorar la diversidad entre poblaciones de maíz en una región: caso la raza Chalqueño. Revista Fitotecnia Mexicana 23:335-354.

Herrera-Cabrera B. E., F. Castillo-González, J. J. Sánchez-González, J. M. Hernández-Casillas, R. Ortega-Paczka y M. M. Goodman (2004) Diversidad del maíz Chalqueño. Agrociencia 38:191-206.

Kuleshov N. N. (1981) Maíces de México, Guatemala, Cuba, Panamá, y Colombia. In: Las Plantas Cultivadas de México, Guatemala y Colombia. S. M. Bukasov (ed). Trad. al español por J. León, de la trad. inglesa de M. H. Byleveld. CATIE. Turrialba, Costa Rica. pp:40-53.
López-Romero G., A. Santacruz-Varela, A. Muñoz-Orozco, F. CastilloGonzález, L. Córdova-Téllez y H. Vaquera-Huerta (2005) Caracterización morfológica de poblaciones nativas de maíz del Istmo de Tehuantepec, México. Interciencia 30:284-290.

Mijangos-Cortés J. O., T. Corona-Torres, D. Espinosa-Victoria, A. Muñoz-Orozco, J. Romero-Peñaloza and A. Santacruz-Varela (2007) Differentiation among maize (Zea mays L.) landraces from the Tarasca Mountain Chain, Mexico and the Chalqueño complex. Genetic Resources and Crop Evolution 54:309-325.

Ortega P. R. y J. J. Sánchez G. (1989) Aportaciones al estudio de la diversidad de maíz de las partes altas de México. Revista Fitotecnia Mexicana 12:105-119.

Pla L. E. (1986) Análisis Multivariado: Métodos de Componentes Principales. Programa Regional de Desarrollo Científico y Tecnológico. Secretaria General de la Organización de los Estados Americanos. Washington, D.C. 94 p.

Rawlings O. J. (1988) Applied Regression Analysis: A Research Tool. Wadsworth \& Brooks/Cole. Advanced Books \& Software. Pacific Grove, California. 553 p.

Rohlf F. J. (2009) NTSYSpc: numerical taxonomy system. Version 2.21c. Exeter Software: Setauket: New York.

Romero P. J., F. Castillo G. y R. Ortega P. (2002) Cruzas de poblaciones nativas de maíz de la raza Chalqueño: II. Grupos genéticos divergencia genética y heterosis. Revista Fitotecnia Mexicana 25:107-115.

Sánchez G. J. J. and M. M. Goodman (1992) Relationships among the Mexican races of maize. Economic Botany 46:72-85.

Sánchez G. J. J., M. M. Goodman and J. O. Rawlings (1993) Appropriate characters for racial classification in maize. Economic Botany 47:44-59.

Sánchez G. J. J., M. M. Goodman and C. W. Stuber (2000) Isozymatic and morphological diversity in the races of maize of Mexico. Economic Botany 54:43-59.

SAS Institute (2002) SAS/STAT User's Guide, Software version 9.0. Cary, N.C., USA. 4424 p.

SMN, Servicio Meteorológico Nacional (2009) ERIC III. Extractor Rápido de Información Climatológica Base CLICOM. V. 2.0. Servicio Meteorológico Nacional. CD Rom. México.

Wellhausen E. J., L. M. Roberts y E. Hernández X. (1951) Razas de Maíz en México, su Origen, Características y Distribución. Folleto Técnico No. 5. Oficina de Estudios Especiales. Secretaría de Agricultura y Ganadería. México, D.F. 237 p. 